\title{
Optimization of AlN thin layers on diamond substrates for high frequency SAW resonators
}

\author{
$\begin{array}{lllll}\text { J.G. Rodríguez-Madrid } \quad \text { G.F. Iriarte } \quad \text { D. Araujo M.P. Villar } & \text { O.A. Williams } \quad \text { W. Müller-Sebert } \quad \text { F. Calle }\end{array}$
}

\begin{abstract}
A B S T R A C T
AIN/diamond heterostructures are very promising for high frequency surface acoustic wave (SAW) resonators. In their design, the thickness of the piezoelectric film is one of the key parameters. On the other hand, the film material quality and, hence, the device performance, also depend on that thickness. In this work, polished microcrystalline diamond substrates have been used to deposit AlN films by reactive sputtering, from $150 \mathrm{~nm}$ up to $3 \mu \mathrm{m}$ thick. A high degree of the $c$-axis orientation has been obtained in all cases. SAW one port resonators at high frequency have been fabricated on these films with a proper combination of the film thickness and transducer size.
\end{abstract}

\section{Introduction}

Day by day the amount of data transmission in mobile phones, satellite services and wireless network systems is increasing. That has resulted in a rising demand in high-performance devices operating in the gigahertz range frequencies. The operating frequency of the surface acoustic wave (SAW) devices is given by $f=v / \lambda$, where $v$ is the phase velocity that depends on the material and $\lambda$ is the wavelength corresponding to the period of the interdigital transducers (IDTs). In order to achieve higher frequencies, either the selection of a suitable material with higher phase velocity or a smaller IDT period can be considered. The combination of both approaches is likely to be necessary for the development of $\mathrm{X}$ band mobile communication systems [1]. Diamond has the highest sound velocity among all materials, about $12,000 \mathrm{~m} / \mathrm{s}[1,2]$. On the other hand, AIN has the highest phase velocity among all piezoelectric materials, about $5600 \mathrm{~m} / \mathrm{s}$. Moreover, the AIN/diamond structure has lower sound velocity dispersion with respect to other piezoelectric films $[1,3]$.

A number of works have been devoted to the fabrication of SAW devices using III-nitrides, like AIN/Si [1,4,5] and GaN/Si [6]. In all cases, the nitride layer was orientated along the $c$ axis. In order to make the most of the AIN/diamond structure and nanolithography advantages, the thickness of the piezoelectric AIN must be optimized [7], not only in terms of crystal quality, but also in enhancing the penetration of the acoustic waves into the fast propagation substrate. However, decreasing the thickness below a certain value hinders the c-axis orientation of the film due to the interface layer [8]. These aspects have been evaluated by the growth of AIN films of different thicknesses on micro crystalline diamond substrates using pulsed DC reactive sputtering at low temperature. The characterization has been done by X-ray diffraction (XRD), atomic force microscopy (AFM), and transmission electron microscopy (TEM). Finally, high frequency devices have been processed to demonstrate the potential of the AIN/diamond structure.

\section{Experimental}

The AlN thin films were deposited in a home built balanced magnetron sputter deposition system on microcrystalline diamond (MCD) substrates. These substrates were fabricated by chemical vapor deposition (CVD) and then polished. The MCD substrates were cleaned using a standard procedure before each deposit: rinsing in pirrolidone for $5 \mathrm{~min}$ at $80^{\circ} \mathrm{C}$, acetone for $5 \mathrm{~min}$ at $60^{\circ} \mathrm{C}$ and methanol in an ultrasonic bath. The sputtering conditions were optimized in order to obtain highly $c$-axis oriented AIN at low temperature, as described elsewhere [8]. AIN films from approximately $150 \mathrm{~nm}$ until $3 \mu \mathrm{m}$ were deposited on microcrystalline diamond substrates under the conditions shown in Table 1.

The structure and morphology of these films were examined by Xray diffraction (XRD) using a Phillips X-Pert Pro MRD diffractometer. Transmission electron microscopy (TEM) analysis in electron diffraction mode was performed on cross-sectional prepared samples using a JEOL 1200 EX electron microscope operating at $120 \mathrm{keV}$. TEM sample preparation was carried out by mechanical cutting, grinding and ion milling to get electron transparency. Micrographs were recorded in bright 
Table 1

Processing parameters for sputtering AlN on diamond substrates.

\begin{tabular}{ll}
\hline Parameter & Value \\
\hline Power [W] & 700 \\
Base pressure [mbar] & $<5 \times 10^{-8}$ \\
Gas composition $\mathrm{Ar} / \mathrm{N}_{2}[\mathrm{sccm}]$ & $3 / 9$ \\
Process pressure $[\mathrm{mTorr}]$ & 3 \\
Substrate temperature $\left[{ }^{\circ} \mathrm{C}\right]$ & 25 \\
Target substrate distance $[\mathrm{mm}]$ & 45 \\
\hline
\end{tabular}

(BF) and dark field (DF) conditions, and also using selective area electron diffraction (SAED).

\section{Results and discussion}

The main difficulties in the synthesis of AIN on diamond are not only due to the intrinsic problems at the interface between the thin layer and the substrate $[1,9]$, but also due to the high roughness of the diamond surface [8]. The surface roughness was analyzed by AFM at different spots within the wafer, as shown in Fig. 1, and the average results were $3.7 \mathrm{~nm}$ root mean square (rms). For this low value, the AIN thin film is well oriented on the c-axis [8]. After the deposition, the average surface roughness of the AIN was $4.2 \mathrm{~nm}$ rms.

Fig. 2 shows the XRD spectra in the $\theta / 2 \theta$ scan mode of several AIN films with different thicknesses deposited on diamond. It can be seen that the AIN films are highly oriented on the $c$-axis, (0002) direction, perpendicular to the surface. The intensity of this peak is reduced when reducing the deposited film thickness. No other diffraction peaks are observed from the AlN film. The substrate grains are randomly oriented and only the diamond (111) direction appears on the spectra.

In order to gage the crystal quality of these AIN films, the rocking curve on the (0002) direction was analyzed. The full width at half maximum (FWHM) corresponding to the (0002)-peak rocking curve is reduced for thicker AIN layers (Fig. 3), down to $2^{\circ}$ for layers thicker than $1 \mu \mathrm{m}$. These results are comparable with that obtained in the literature $[8,10,11]$. Therefore, the crystal quality for the sputtered AIN films on diamond improves as its thickness increases, up to a saturation value.

TEM analysis has been done to confirm these results and to understand them from a structural point of view. Fig. 4 shows the columnar growth of the AIN on the diamond substrate observed by TEM, with three regions of the AIN layer grown directly on the diamond substrate recorded in $\mathrm{BF}$ conditions. The respective insets correspond to

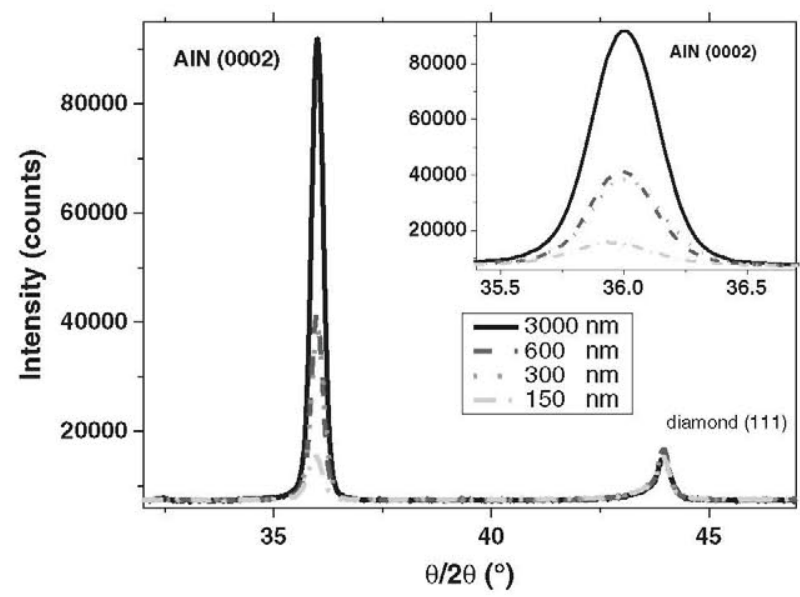

Fig. 2. Influence of the AlN layer thickness on the $\theta / 2 \theta$ XRD-spectra and 0002-rocking curves (inset).

the electron diffraction of the regions indicated by the dashed circles, for which the micrographs were recorded using the selective area aperture. The aperture of locations 1, 2 and 3 is located at the bottom, the center and the top of the AIN layer, respectively. The corresponding SAED patterns show that the spots gradually change from circular shape to a well defined spot. This indicates that the grain disorientation is reduced with the layer thickness. Near the interface, the grains have an estimated misorientation of around $20^{\circ}$, while in the center of the layer this value is reduced to $6^{\circ}$, and at the top of the layer grains are almost perfectly oriented. These results agree with those obtained by X-ray diffraction: for thinner AIN layers, a larger FWHM of the 0002-rocking curve is observed, in other words, the grain misorientation increases.

A more accurate analysis of the SAED of location 3 shows that it corresponds to two superimposed patterns: (i) one spot system corresponding to the $\left[\begin{array}{lll}2 & 1 & 10\end{array}\right]$ pole (i.e. crystal oriented along this direction), (ii) and the other parallel to the [01 10] direction, as shown in Fig. 4 by the rectangles in the inset of location 3 . The orientation of these two patterns corresponding to the two columnar grain families has the same $z$-orientation, i.e. the grains are rotated around this z-axis.

The layers have been used to fabricate high frequency one-port SAW devices. Fig. 5 shows the frequency response obtained for a device processed on $600 \mathrm{~nm}$ thick AIN layer deposited on $8 \mu \mathrm{m}$ of MCD, with a rocking curve FWHM of $3.3^{\circ}$. The IDT wavelength is $\lambda=800 \mathrm{~nm}$. Three resonant peaks can be observed in the response. The first peak
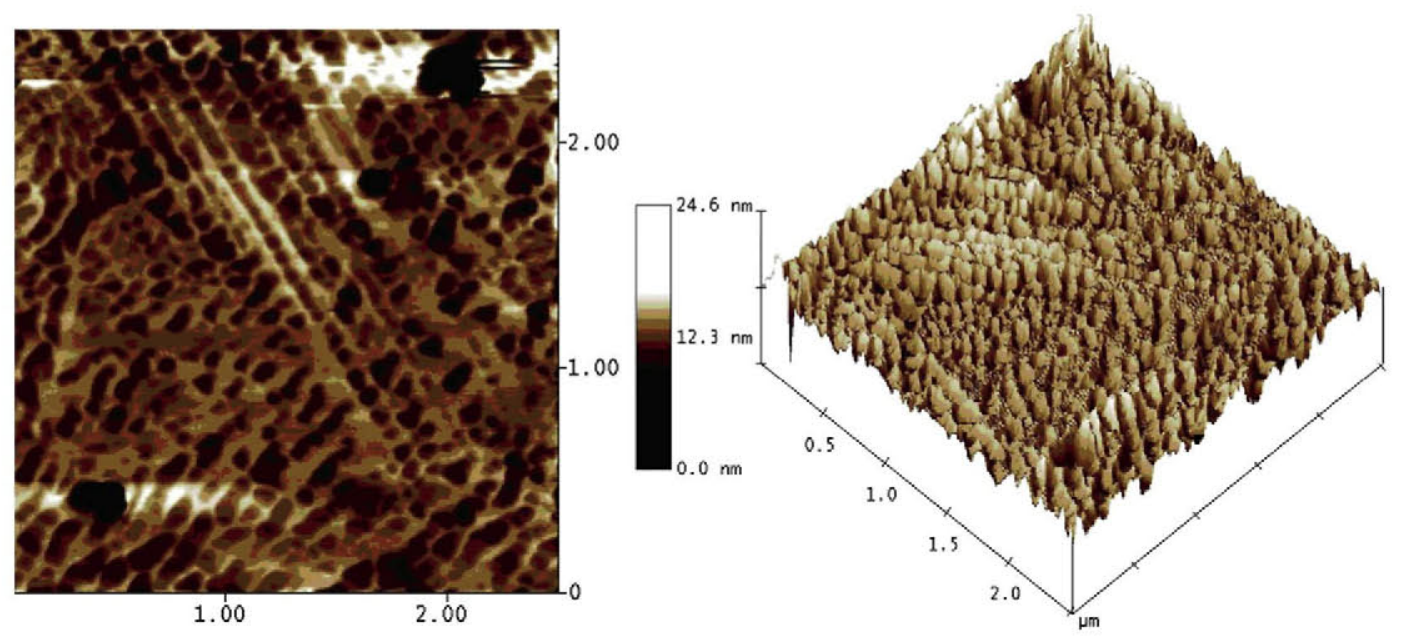

Fig. 1. Height (left) and 3D (right) AFM micrographs of a microcrystalline diamond wafer. 


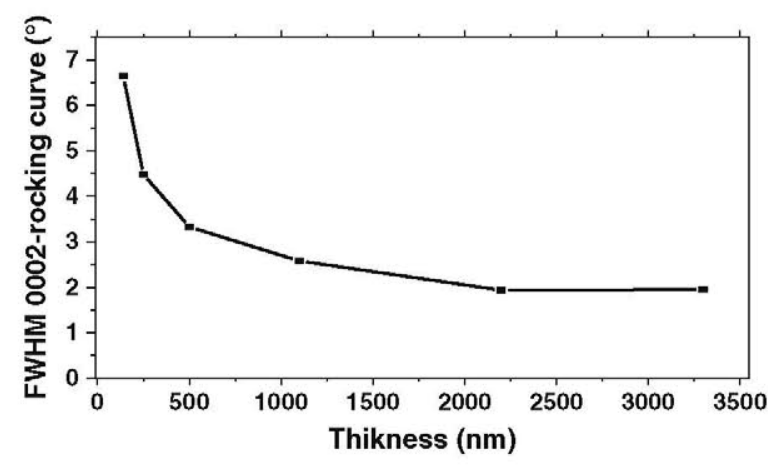

Fig. 3. Influence of the AIN layer thickness on the FWHM 0002-rocking curve.

at a frequency of $10.97 \mathrm{GHz}$ corresponds to the first Sezawa SAW mode [10], at Vp around $8776 \mathrm{~m} / \mathrm{s}$, higher than the sound velocity for the AIN due to wave propagation through the substrate. The other peaks, at $13.06 \mathrm{GHz}$ and $14.34 \mathrm{GHz}$, correspond to second and third order Sezawa SAW modes. So far, these frequencies are the highest obtained for SAW AIN/diamond devices $[5,6,10]$. The appropriate thickness of the piezoelectric film in the AlN/diamond structure, combined with nanolithography, may allow to increase the frequency over $40 \mathrm{GHz}$.

\section{Conclusions}

In conclusion, the synthesis of AIN by reactive sputtering on diamond substrates has been optimized for different film thicknesses,

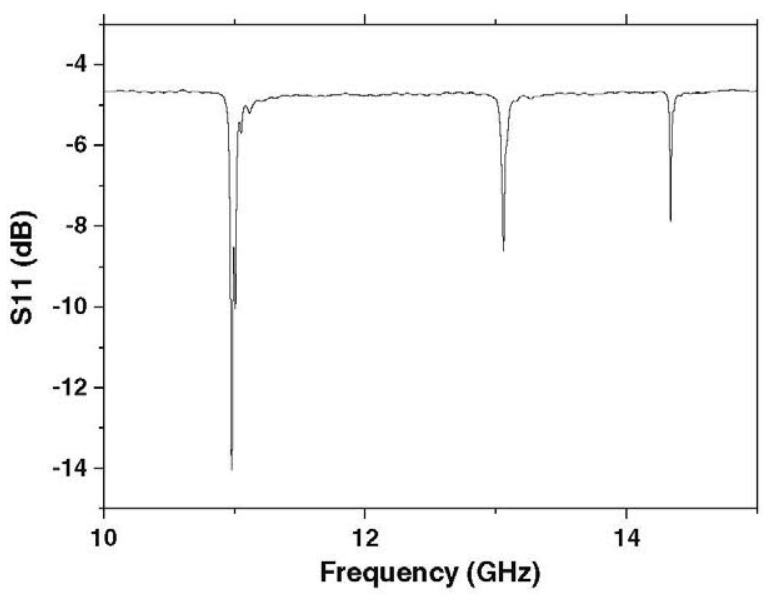

Fig. 5. Reflection coefficient of an AlN/diamond SAW resonator with $10.97 \mathrm{GHz}$ central frequency.

in order to fabricate SAW devices for high frequency applications. When the thickness increases, the orientation on the (0002) direction improves, as proved by X-ray diffraction and TEM analysis. Grown on diamond substrates at room temperature, a very high degree of $c$-axis orientation of the AIN films is shown, even for the smallest thickness. These layers were used to process one port SAW resonators operating at frequencies above $10 \mathrm{GHz}$.

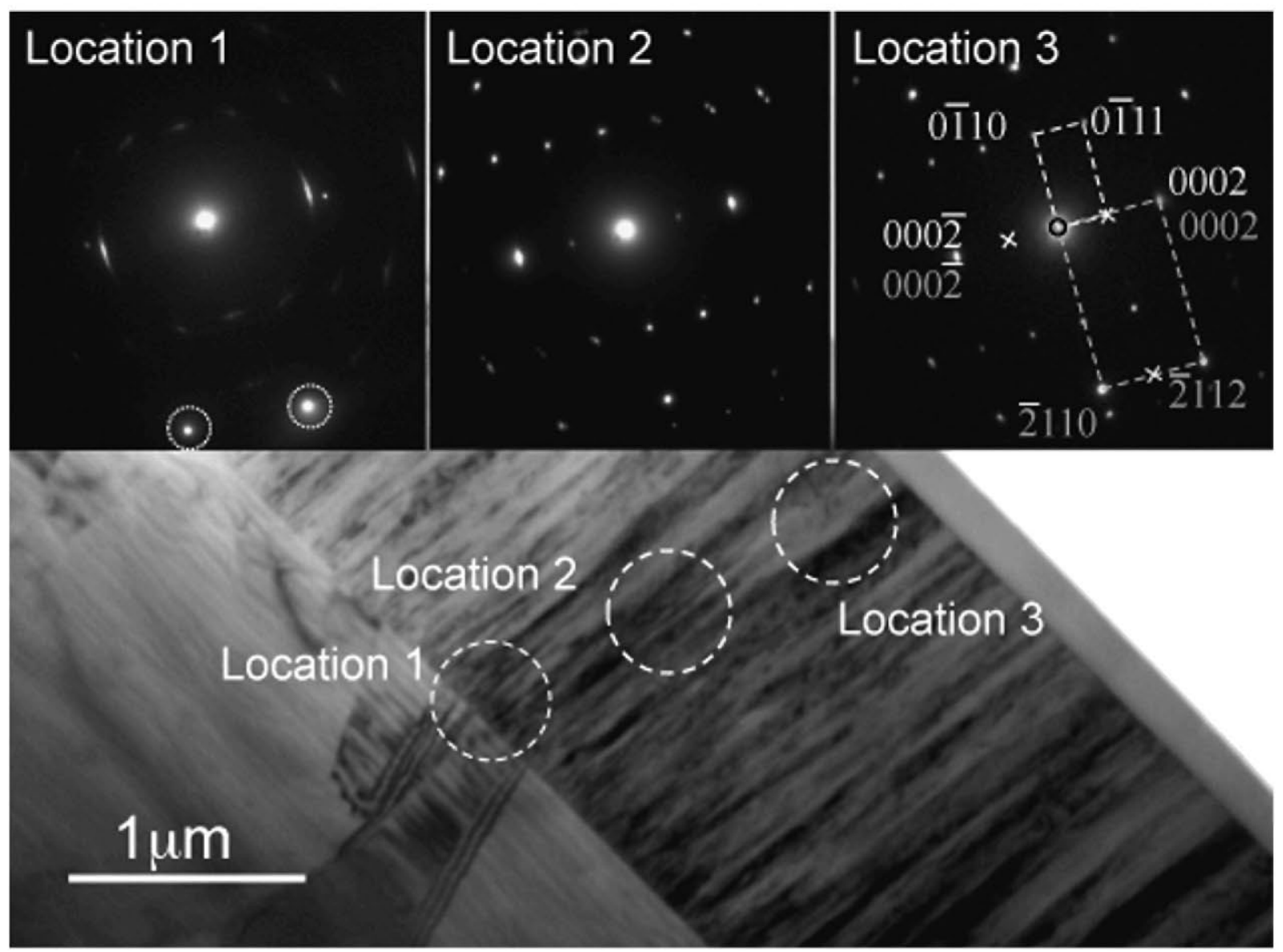

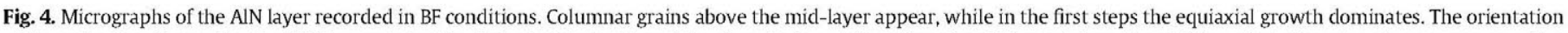

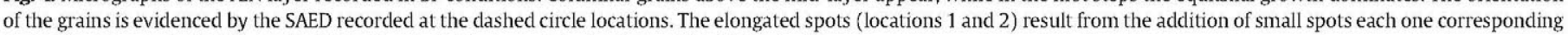
to a grain. Two grain families with different orientations are detected in location 3 (see text). 


\section{Acknowledgments}

The authors would like to acknowledge the support of this work by Ministerio de Ciencia e Innovación, under the MINANI (TEC200767065/MIC) and ReADi (TEC2010-19511) projects, and the Acción Integrada DE2009-0015.

\section{References}

[1] Assouar MB, Elmazria O, Kirsch P, Alnot P, Mortet V, Tiusan C. High-frequency surface acoustic wave devices based on AlN/diamond layered structure realized using e-beam lithography. J Appl Phys 2007;101:114507.

[2] Ishihara M, Nakamura T, Kokai F, Koga Y. Preparation of AlN and LiNbO3 thin films on diamond substrates by sputtering method. Diamond Relat Mater 2002;11: 408-12.

[3] Benetti M, Cannatà D, Di Pietrantonio F, Verona E, Generosi A, Paci B, et al. Growth and characterization of piezoelectric AlN thin films for diamond-based surface acoustic wave devices. Thin Solid Films 2006;497:304-8.
[4] Kirsch P, Assouar MB, Elmazria O Mortet V, Alnot P. $5 \mathrm{GHz}$ surface acoustic wave devices based on aluminum nitride/diamond layered structure realized using electron beam lithography. Appl Phys Lett 2006;88:223504.

[5] Neculoiu D, Müller A, Deligeorgis G, Dinescu A, Stavrinidis A, Vasilache D, et al. AIN on silicon based surface acoustic wave resonators operating at $5 \mathrm{GHz}$. Electron Lett 2009;45:1196.

[6] Müller A, Neculoiu D, Konstantinidis G, Deligeorgis G, Dinescu A, Stavrinidis A et al. SAW devices manufactured on $\mathrm{GaN} / \mathrm{Si}$ for frequencies beyond $5 \mathrm{GHz}$. IEEE Electron Device Lett 2010;31:12.

[7] Benetti M, Cannata D, Di Pictrantonio F, Verona E. Growth of AlN piezoelectric film on diamond for high-frequency surface acoustic wave devices. IEEE Trans Ultrason Ferroelectr Freq Control 2005;52:1806-11.

[8] Iriarte GF, Rodriguez JG, Calle F. Synthesis of c-axis oriented AlN thin films on different substrates: a review. Mater Res Bull 2010;45:1039-45.

[9] Chou C, Lin Y, Huang J, Tai NH, Lin I. Growth of high quality AlN thin films on diamond using TiN/Ti buffer layer. Diamond Relat Mater 2006;15:404-9.

[10] Iriarte GF. Surface acoustic wave propagation characteristics of aluminum nitride thin films grown on polycrystalline diamond. J Appl Phys 2003;93:9604-9.

[11] Chou C, Lin Y, Huang J, Tai NH, Lin I. Growth of high quality AlN thin films on diamond using TiN/Ti buffer layer. Diamond Relat Mater 2006;15:404-9. 